Original Paper

\title{
Bright-Dark Rogue Waves
}

\author{
Hani Kbashi ${ }^{1}$, Sergey V. Sergeyev ${ }^{1 *}$, Chengbo Mou ${ }^{2 *}$, Amos Martinez Garcia ${ }^{1}$, \\ Mohammed Al Araimi ${ }^{1,3}$, Aleksei Rozhin ${ }^{1}$, Stanislav Kolpakov ${ }^{1}$, and Vladimir
}

Kalashnikov ${ }^{4}$

*Corresponding Authors: E-mails: s.sergeyev@aston.ac.uk, mouc1@ @ shu.edu.cn

${ }^{1}$ Aston Institute of Photonic Technologies, Aston University, Aston Triangle, B4 7ET, Birmingham, United Kingdom

${ }^{2}$ Key Laboratory of Specialty Fiber Optics and Optical Access Networks, Shanghai

University, 200072, Shanghai, China

${ }^{3}$ Engineering Department, Al Musanna College of Technology, Muladdah Musanna, P.O. Box 191, P.C. 314, Sultanate of Oman

${ }^{4}$ Institute of Photonics, Vienna University of Technology, Gusshausstr. 27/387, Vienna, A-1040, Austria

During the last two decades, revealing mechanisms of origin waves with anomalous amplitude (rogue waves) is in focus of researchers from different fields ranging from oceanography to the laser physics. Mode-locked laser, as a test bed system, provide a unique opportunity to collect more data on rogue waves in the form of random pulses (soliton rain) and to clarify mechanisms of rogue wave emergence caused by soliton-soliton and soliton-dispersive wave interactions. Here, for the first time, it is demonstrated experimentally and theoretically for Er-doped modelocked laser a new type of vector rogue waves driven by desynchronization of the orthogonal linear states of polarization and so leading to the output power oscillations in the form of anomalous spikes-dips (bright-dark rogue waves). Results can pave the way to the unlocking the universal nature of the origin of the rogue waves and so can be of interest to a broad scientific community.

\section{Introduction}

Observation of extreme events (rogue waves, RWs) with the unpredictable nature of emergence and disappearance has been initially reported in oceanography [1] and further in different fields ranging from the financial markets [2] to nonlinear optics and laser physics [3-14]. To satisfy RWs criteria, extreme events should arise more frequently than events satisfying Gaussian or Rayleigh distributions and should have amplitudes more than twice as large as the significant 


\section{WILEY-VCH}

wave height (SWH), i.e. the mean amplitude of the highest third of the waves [1-3]. At present, it is more common to use the other criteria of the RWs where the RW's amplitude should exceed the standard deviation of the ocean's surface variations in more than eight times [3].

The major obstacle for studying rogue waves in the context of prediction and mitigation are the scarcity of these rare events and the inability to perform full-scale experiments on RWs emergence in real-world scenarios. Mode-locked fiber lasers (MLFLs) as a source of pico- and femtosecond pulses with $\mathrm{MHz}$ repetition rates provide a unique opportunity to observe more data on rogue waves in the short time (compared to the time scale of RWs in other systems, such as in the ocean and financial market [1-3] ) and under laboratory-controlled conditions [712]. Previously it has been found that RW can be generated in mode-locked lasers at the time scale of round trip [8-12] because of soliton-soliton interaction through the overlapping of their tails or soliton-dispersive wave interaction $[15,16]$. The result of these interactions is the coupling enhancement that leads to chaotic pulse bunching (emerging soliton rain) at the time scale of a round-trip time [7-12].

All of the above experimental observations report the existence of either bright or dark rogue waves [3-13]. The co-existence of the bright-dark rogue waves (BDRWs) has been predicted theoretically using coupled nonlinear Schrödinger equation (NLSE) systems [13, 14], but has never been observed experimentally in optics. In this Article, for the first time, it is demonstrated experimentally and theoretically for Erbium-doped laser mode-locked with carbon nanotubes an additional to soliton-soliton and soliton-dispersive wave interaction mechanism of the RWs emergence. This mechanism leading to emergence of BDRWs is caused by desynchronization of the linear states of polarizations (SOPs) that is justified with the help of new vector model which is different from the previously developed models based on coupled Schrödinger or Ginzburg-Landau equations $[13,14]$. 


\section{WILEY-VCH}

\section{Experimental results on bright-dark rogue waves characterization}

\subsection{The experimental set-up.}

The laser (Fig. 1) comprises 1.1m long Erbium-doped fiber (EDF) with a nominal absorption ratio of $80 \mathrm{~dB} / \mathrm{m}$ at $1530 \mathrm{~nm}$. The group velocity dispersion of the EDF is of $+59 \mathrm{ps}^{2} / \mathrm{km}$. A fiber pigtailed optical isolator (OISO) has been used to support a unidirectional operation of the laser. A $975 \mathrm{~nm}$ laser diode (LD) with the maximum output power of $200 \mathrm{~mW}$ is used to pump the laser via a 980/1550 wavelength division multiplexer (WDM). A standard 70:30 fused coupler (OUTPUT C) has been installed to redirect of $30 \%$ of the laser light out of the cavity. The rest of the laser cavity includes $1.22 \mathrm{~m}$ of OFS 980 fiber and $4.4 \mathrm{~m}$ of SMF 28 fiber. The CNT modelocker is used in the form of a piece of the carbon nanotube polymer composite film placed between two standard fiber connectors supplemented by the index matching for minimization of insertion losses. The total cavity GVD is of $-0.04 \mathrm{ps}^{2} / \mathrm{nm}$.

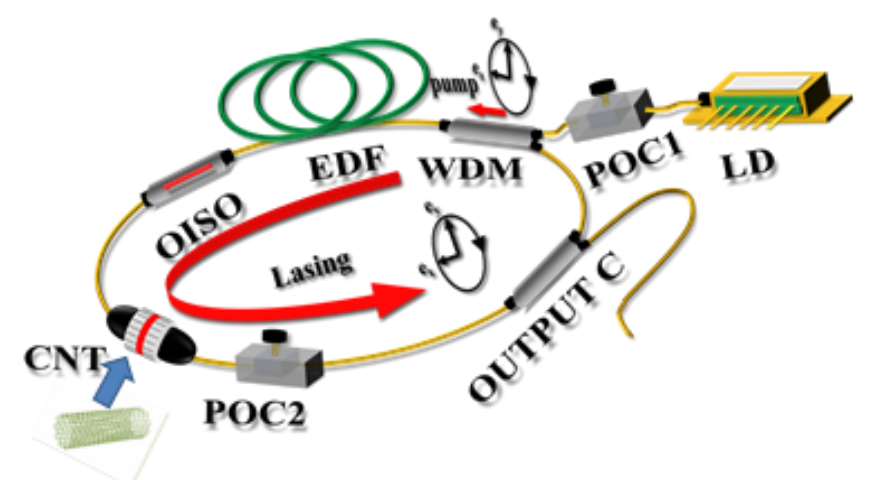

Figure 1 Schematic of a fiber laser mode-locked with carbon nanotubes: Erbium-doped fiber (EDF); optical isolator (OISO); laser diode (LD); 980/1550 wavelength division multiplexer (WDM); the carbon nanotubes-based mode locker (CNT); polarization controllers (POC1 and POC2); output coupler (output C).

Two polarization controllers (POC1 and POC2) are installed to adjust the SOP of the pump wave and the in-cavity birefringence. The polarimeter (Thorlabs IPM5300, $1 \mu$ s resolution, 1024 samples) has been connected to the output. To obtain results shown in Figs. 2, the laser signal is measured by using a $U D P-15-I R-2 F C$ detector with a bandwidth of $17 \mathrm{GHz}$; the electric signal from the detector has been recorded by a Tektronix DPO7254-oscilloscope (with 25 ps sample interval and $20 \mathrm{~K}$ in each oscillogram). The oscilloscope data comprise 11 


\section{WILEY-VCH}

oscillograms of the pulse dynamics with $20 \mathrm{~K}$ points in each oscillogram. To obtain results shown in Fig. 4, the output light from the laser cavity is detected using a 50-GHz photodetector (Finisar XPDV2320R) and recorded by 32 GHz sampling oscilloscope (Agilent DSOX93204A). We have recorded oscilloscope traces for $26 \mathrm{~ms}$ with an effective resolution of 12.5 ps per point and 8 bits of the bits of resolution. To analyze the results using the spatiotemporal dynamics, we split the $26 \mathrm{~ms}$ oscilloscope traces into segments. The length of the segment is equal to the one round trip. Then these segments were processed as a matrix which providing the intensity evolution through ten thousand of round trips. The POC1 and POC2 have been adjusted to find conditions for RWs emergence. The optical spectrum has been measured with the optical spectrum analyzer (ANDO AQ6317B) and the pulse width - with the help of the autocorrelator (Pulsecheck), autocorrelation data consist of 8 traces, and polarimetry data include 20 traces.

\subsection{Experimental observation of bright-dark rogue waves driven by soliton-soliton interaction and the desynchronization of the orthogonal linear polarized SOPs.}

The obtained results are shown in Figs. 2 (a-d) for the pump powers $\mathrm{P}=26.4 \mathrm{~mW}$ and $\mathrm{P}=32 \mathrm{~mW}$. As follows from Fig. 2 (a), multi-pulse dynamics is similar to the soliton rain with the main difference in low pump power of $26.4 \mathrm{~mW}$ used here as compared to $800 \mathrm{~mW}$ used by the other authors [8-12]. When pump power is increased to $32 \mathrm{~mW}$, the number of pulses in a bunch is increasing (Fig. 2 (b)). As follows from Fig. 1 (a, b), the dynamics is stable within each slice of 16 round-trips and is changing from slice to slice. Probability distribution histograms are shown in Fig. 1 (c, d). In view of each output voltage $\mathrm{V}$ is normalized as $\mathrm{V}_{\mathrm{n}}=(\mathrm{V}$-median $(\mathrm{V})) / \sigma(\mathrm{V})$, the $\mathrm{RW}$ criterion looks as $\mathrm{V}_{\mathrm{n}}>8$ and so both oscillograms in Fig. 1 (a, b) demonstrate the presence of bright rogue waves (BRWs). Soliton-soliton interaction leading to the BRWs appearance on the scale of few round-trips is discussed in Supporting Information. The total number of round-trips measured by oscilloscope is 171 that is not enough to apply a low pass filter to filter out such interactions.

To get insight into the mechanism of the RW emergence at the slow time scales of $1 \mu \mathrm{s}-20$ $\mathrm{ms}$ (660K round-trips) and so to eliminate the soliton-soliton and soliton-dissipative wave interactions, we combined 20 slices of the temporal waveforms obtained with the help of a polarimeter. We measured the normalized Stokes parameters $s_{1}, s_{2}, s_{3}$ which are related to the 


\section{WILEY-VCH}

output powers of two linearly cross-polarized SOPs $I_{x}$ and $I_{y}$, and the phase difference between them $\Delta \phi$ as follows:

$$
S_{0}=I_{x}+I_{y}, S_{1}=I_{x}-I_{y}, S_{2}=2 \sqrt{I_{x} I_{y}} \cos \phi, S_{3}=2 \sqrt{I_{x} I_{y}} \sin \Delta \phi, s_{i}=\frac{S_{i}}{\sqrt{S_{1}^{2}+S_{2}^{2}+S_{3}^{2}}},(i=1,2,3)
$$

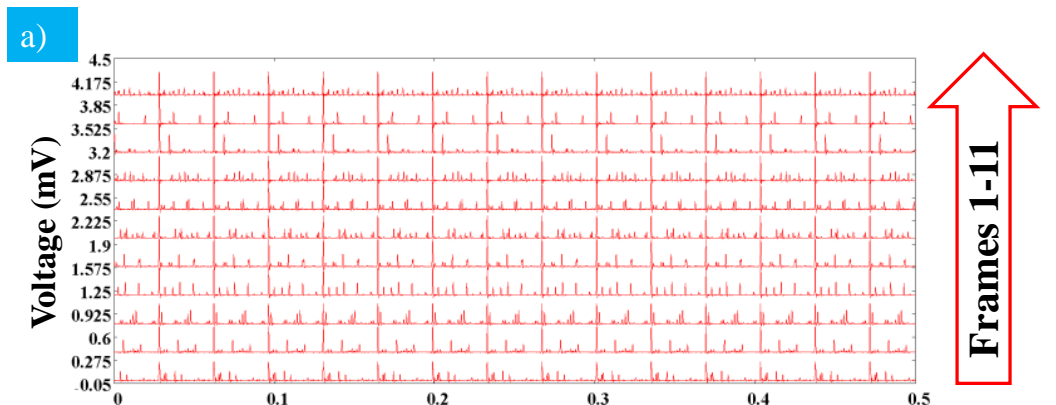

$$
\text { b) }
$$

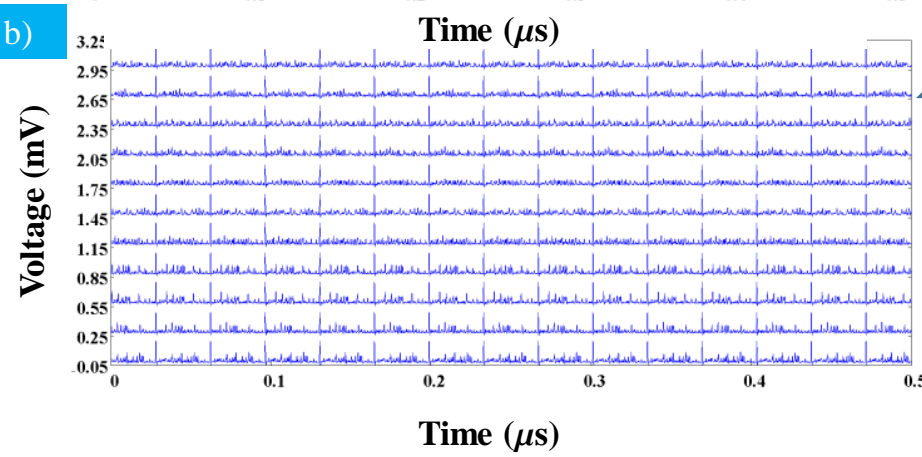

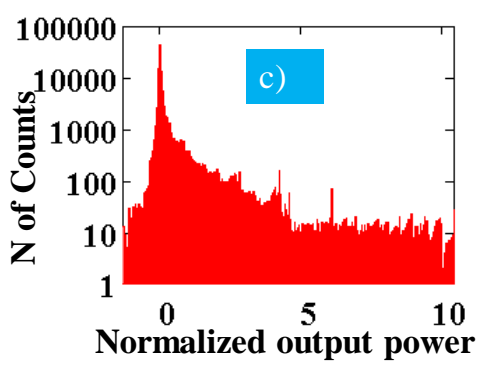

(a.u.)
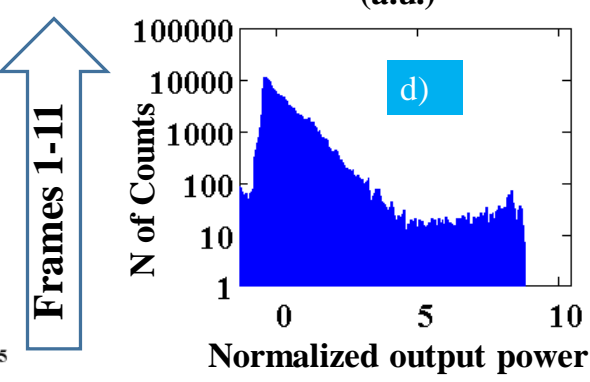

(a.u.)

Figure 2 Bright rogue waves at the time scale of round-trip time. a), b) 11 slices of the oscillogram (25 ps sample interval, 16 round trips per slice; in view of voltage offset in the oscilloscope, measured minimum voltage can be negative); c), d) probability distribution histograms (each output voltage $\mathrm{V}$ is normalized as : $\mathrm{V}_{\mathrm{n}}=(\mathrm{V}$-median $(\mathrm{V})) / \sigma(\mathrm{V})$; RW criterion is $\mathrm{V}_{\mathrm{n}}>8$. Parameters: a), c) $\mathrm{P}=26.4 \mathrm{~mW}$; b), d) $\mathrm{P}=32 \mathrm{~mW}$.

Results are shown in Fig. 3 for the pump current setting corresponding to the Fig. 2 (a-d). As follows from Fig. 3 (a1) for $\mathrm{P}=26.4 \mathrm{~mW}$, the anomalous spikes in the output power at the slow time scale correspond to the phase difference jumps in $\pi$, i.e. transitions between orthogonally polarized SOPs as shown in Fig. 3 (a1, b1). After setting pump power as $\mathrm{P}=32 \mathrm{~mW}$ and tuning POC2, the output power is oscillating without the presence of RW events (Fig. 2 (a2)). As shown in Fig. 2 (a2, b2), oscillating phase difference (between 0 and $\pi$ ) rather than randomly evolving is a signature of an absence of RWs events at the slow time scale (Fig.2 (c2)). 


\section{WILEY-VCH}

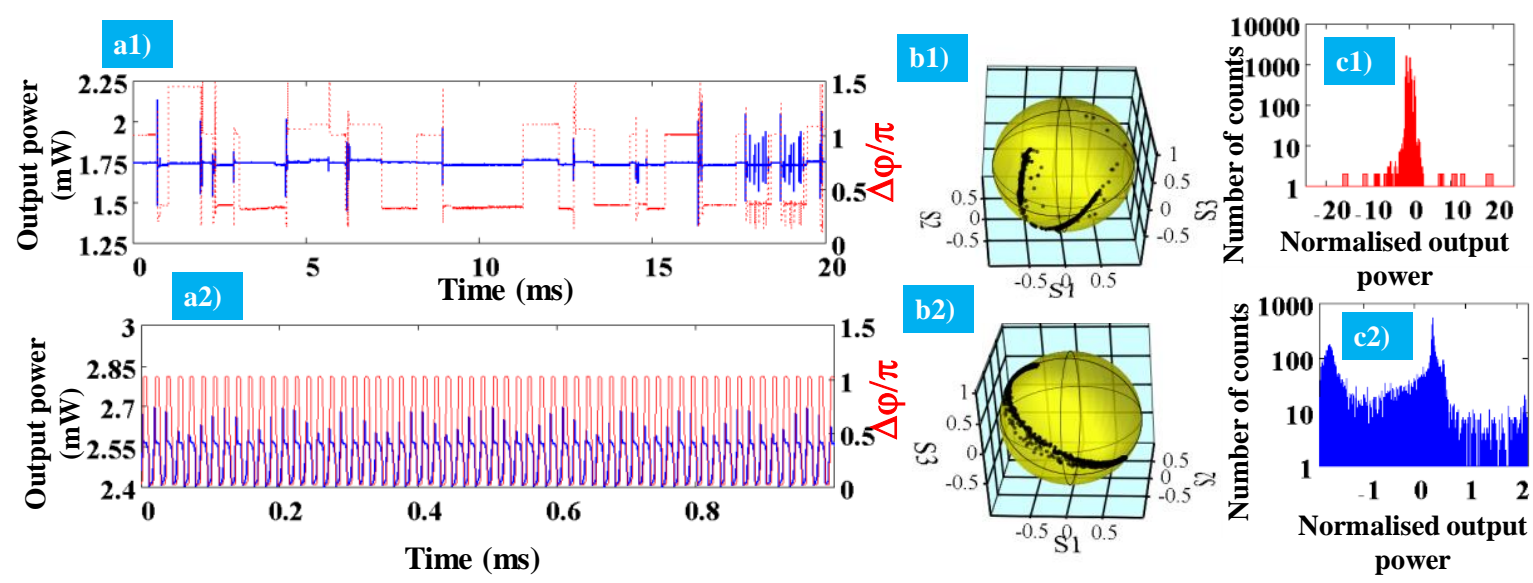

Figure 3 Polarization laser dynamics at the time scale of tens of thousands of round trips. (a) $-\mathrm{c})(1 \mu \mathrm{s}$ resolution, i.e. averaging over approximately 33 round trips, 20 slices with 1024 points per slice): a1) a2) the output powers $\mathrm{I}=\mathrm{I}_{\mathrm{x}}+\mathrm{I}_{\mathrm{y}}$ (blue) and the phase difference (red) vs time; b1), b2) trajectories in normalized Poincaré sphere; c1), c2) probability distribution histograms for the total output power $\mathrm{I}=\mathrm{I}_{\mathrm{x}}+\mathrm{I}_{\mathrm{y}}$ (bright-dark RWs in Fig. 2 (c), the absence of RWs in Fig. 2 (d) ). Each output power is normalized as shown in Fig.2. Parameters: a1) - c1) P=26.4 mA; a2)-c2) P=32 mW.

Probability distribution histograms for the total output power $\mathrm{I}=\mathrm{I}_{\mathrm{x}}+\mathrm{I}_{\mathrm{y}}$ are shown in Fig. 3 (c1, c2). As follows from Fig. 3 (c1), the presence of so-called "bright-dark rogue waves" (spikes and dips [13, 14]) is caused by the interaction of two orthogonal SOPs. Interaction of the orthogonal SOP leads to the "antiphase" dynamics of the output powers $\mathrm{I}_{\mathrm{x}}$ and $\mathrm{I}_{\mathrm{y}}$ and so to the BDRWs emergence during the phase difference jumps in $\pi$ (Fig. 3 (a1)). As follows from the Fig. 3 (c2), there is no RW in the case of regular phase difference oscillations between 0 and $\pi$ (Fig. 3 (a2, b2)).

\section{3 Experimental observation of bright-dark rogue waves driven by the desynchronization}

\section{of the orthogonal linear polarized SOPs.}

To eliminate the soliton-soliton and soliton-dissipative wave interactions at the fast (round-trip time) scale, we decreased the pump power $\mathrm{P}=18.4 \mathrm{~mW}$ and tuned $\mathrm{POC} 1$ and $\mathrm{POC} 2$ to suppress soliton rain. As a result, we observed the dark-bright rogue waves as shown in Fig.4 (a-d). Unlike the previous cases shown in Figs. 2 and 3, the output power is randomly changing from pulse to pulse (Fig. 4 (a, d) ) with the statistics satisfying the bright-dark rogue waves emergence (Fig. 4 (c)). The data obtained with the help of polarimeter are shown in Fig. 5 (a-c). The same way as in Fig. 3, the anomalous spikes in the output power satisfying RWs criteria follow the phase difference jumps in $\pi$, i.e. transitions between orthogonally polarized SOPs (Fig. $5(\mathrm{a}, \mathrm{b})$ ). 


\section{WILEY-VCH}

Thus, the experimental data demonstrate three different cases for RWs appearance at the fast (round trip scale) and slow (tens of thousands of round trips) time scales: (i) soliton rain (fast
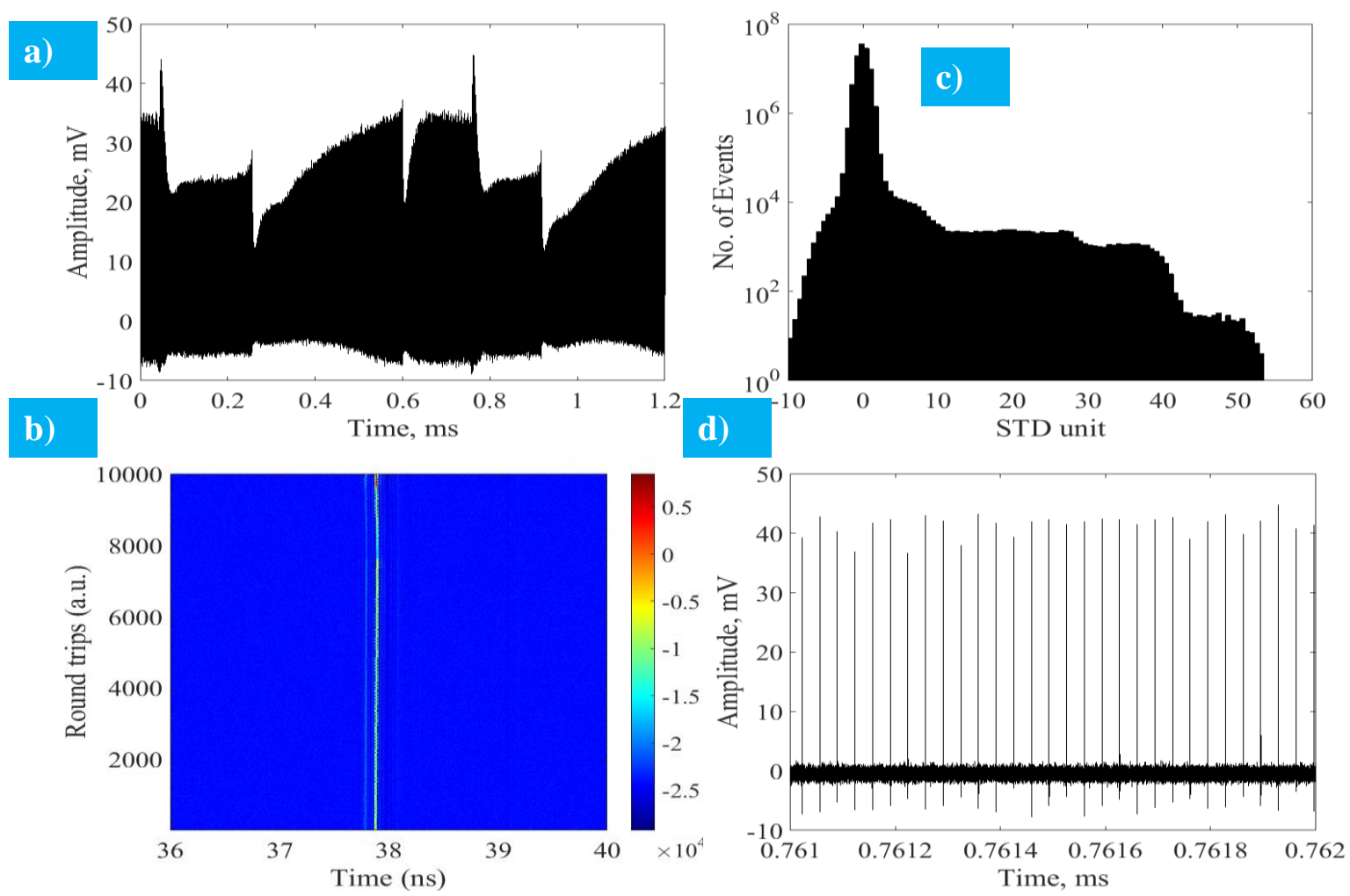

Figure 4 Laser dynamics demonstrating the emergence of the bright-dark rogue waves at pump power of $18.4 \mathrm{~mW}$. (a) oscilloscope traces (32 GHz resolution); (b) Spatio-temporal dynamics (roundtrip time vs a number of round trips); (c) PDF histogram; d) part of the oscillogram demonstrating the absence of the soliton rain.
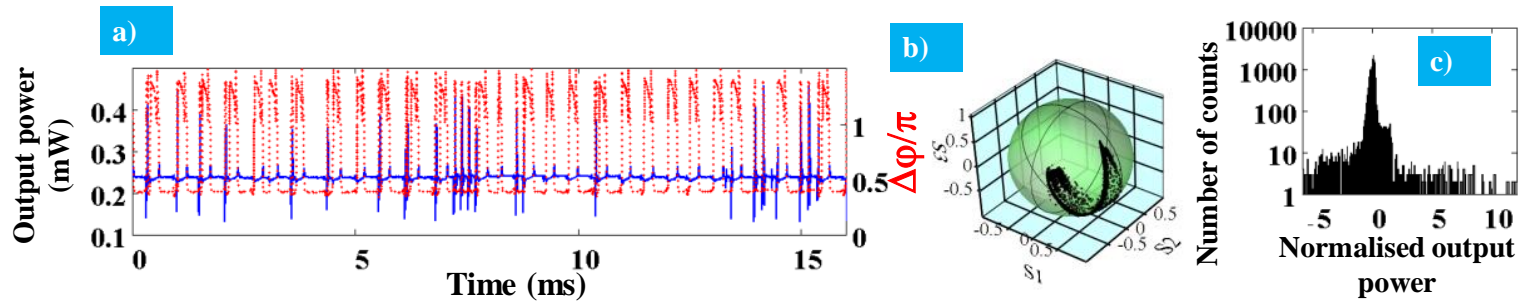

Figure 5 Polarization laser dynamics at the time scale of tens of thousands of round trips. a) - c) polarization measurements ( $1 \mu$ s resolution, i.e. averaging over approximately 33 round trips, 16 slices with 1024 points per slice): a) the output power vs time (blue) and the phase differences (red); and b) trajectories in normalized Poincaré sphere. c) Probability distribution histogram for the total output power $I=I_{x}+I_{y}$. The output power I is normalized as shown in Fig.1. Parameters: a) $\left.-c\right)$ P=18.4 mW.

time scale) and "bright" and "dark" RWs (slow time scale) for $\mathrm{P}=26.4 \mathrm{~mW}$, (ii) soliton rain (fast time scale) and no RWs (slow time scale) for $\mathrm{P}=32 \mathrm{~mW}$, and (iii) the absence of the soliton 


\section{WILEY-VCH}

rain and the presence of RWs at the fast and slow time scales for $\mathrm{P}=18.4 \mathrm{~mW}$. As known from literature [10], dispersive waves produce spectral sidebands that are clearly visible on the optical spectrum. In our case (Fig. S1 in the Supporting Information), we have a smooth optical spectrum without sidebands that indicate that contribution of the soliton-dispersive wave interaction cannot be identified.

\section{Theoretical analysis}

To justify mechanism of the RWs emergence at the fast and slow time scales, we use our recently developed vector model of Erbium-doped fiber laser mode-locked with CNT [17, 18]. The model describes the evolution of the lasing field for two orthogonal SOPs $u=I_{x}^{1 / 2} \exp \left(i \phi_{x}\right), u=I_{y}^{1 / 2} \exp \left(i \phi_{y}\right)$ averaged over the pulse width and the population of the first excited level in the $\mathrm{Er}^{3+}$ doped active medium $[17,18]$. We introduced a slow-time variable $t_{s}=z /\left(V_{g} t_{R}\right)$, where $t_{R}=L / V_{g}$ is the photon roundtrip time, $L$ is the cavity length) and assumed an ansatz in the form $E_{x}\left(t, t_{s}\right)=u\left(t_{s}\right) \operatorname{sech}\left(t / T_{p}\right)$, $E_{y}\left(t, t_{s}\right)=v\left(t_{s}\right) \operatorname{sech}\left(t / T_{p}\right), \quad\left(T_{p}\right.$ is the pulse width). After averaging over the time $T_{p}<<t<<t_{R}$ we obtained the following equations $[17,18]$ :

$$
\begin{aligned}
& \frac{d|u|}{d t}=\left(\frac{\alpha_{1}\left(f_{1}+f_{2}\right)}{1+\Delta^{2}}-\alpha_{2}-F(|u|,|v|)|u|+\frac{\alpha_{1} f_{3}}{1+\Delta^{2}}|v| \cos (\Delta \phi)-\frac{\alpha_{1} f_{3} \Delta}{1+\Delta^{2}}|v| \sin (\Delta \phi)+\frac{2 \gamma}{3}|v|^{2}|u| \sin (2 \Delta \phi),\right. \\
& \frac{d|v|}{d t}=\left(\frac{\alpha_{1}\left(f_{1}-f_{2}\right)}{1+\Delta^{2}}-\alpha_{2}-F(|u|,|v|)|v|+\frac{\alpha_{1} f_{3}}{1+\Delta^{2}}|u| \cos (\Delta \phi)+\frac{\alpha_{1} f_{3} \Delta}{1+\Delta^{2}}|u| \sin (\Delta \phi)-\frac{2 \gamma}{3}|u|^{2}|v| \sin (2 \Delta \phi)\right. \text {, } \\
& \frac{d \Delta \phi}{d t}=\Delta \Omega+K_{N L} \sin ^{2}(\Delta \phi)-K \sin (\Delta \phi-\alpha) \text {, } \\
& \frac{d f_{1}}{d t}=\varepsilon\left[\frac{(\chi-1) I_{p}}{2}-1-\left(1+\frac{I_{p}}{2}+d_{1} S_{0}\right) f_{1}-\left(d_{1} S_{1}+\frac{I_{p}}{2} \frac{\left(1-\delta^{2}\right)}{\left(1+\delta^{2}\right)}\right) f_{2}-d_{1} S_{2} f_{3}\right], \\
& \frac{d f_{2}}{d t}=\varepsilon\left[\frac{\left(1-\delta^{2}\right)}{\left(1+\delta^{2}\right)} \frac{I_{p}(\chi-1)}{4}-\left(\frac{I_{p}}{2}+1+d_{1} S_{0}\right) f_{2}-\left(\frac{\left(1-\delta^{2}\right)}{\left(1+\delta^{2}\right)} \frac{I_{p}}{2}+d_{1} S_{1}\right) \frac{f_{1}}{2}\right], \\
& \frac{d f_{3}}{d t}=-\varepsilon\left[\frac{d_{1} S_{2} f_{1}}{2}+\left(\frac{I_{p}}{2}+1+d_{1} S_{0}\right) f_{3}\right] \text {, } \\
& \Delta \Omega=\frac{2 \alpha_{1} f_{2} \Delta}{1+\Delta^{2}}-2 \beta, K_{N L}=\frac{\gamma L I_{s s}}{6}\left(\left.v\right|^{2}-|u|^{2}\right), K=\frac{4 \alpha_{1} f_{3}}{\left(1+\Delta^{2}\right)}, \operatorname{tg}(\alpha)=\frac{\left(\left.v\right|^{2}-|u|^{2}\right) \Delta}{|u|^{2}+|v|^{2}}, \\
& F(|u|,|v|)=\frac{\alpha_{0}}{\pi}\left(1-\sqrt{\frac{\alpha_{s}\left(|u|^{2}+|v|^{2}\right)}{1+\alpha_{s}\left(|u|^{2}+|v|^{2}\right)}} \arctan \left(\sqrt{\left.\frac{\alpha_{s}\left(|u|^{2}+|v|^{2}\right)}{1+\alpha_{s}\left(|u|^{2}+|v|^{2}\right.}\right)}\right)\right) .
\end{aligned}
$$

Here time is normalized to the round trip time; pump and lasing powers $\left(I_{p}\right.$ and $\left./ u / 2,{ }^{2} / 2\right)$ are normalized to the corresponding saturation powers $I_{p s}$ and $I_{s s}[17,18] ; \alpha_{1}$ is the total absorption of Erbium ions at the lasing wavelength, $\alpha_{0}$ is CNT absorption coefficients at the lasing wavelength, $\alpha_{3}$ is the ratio of saturation powers for CNT and Erbium-doped fiber, $\alpha_{2}$ is the total 


\section{WILEY-VCH}

insertion losses in cavity, $\beta$ is the birefringence strength $\left(2 \beta=2 \pi L / L_{b}, L_{b}\right.$ is the beat length); $\gamma=\gamma_{k} L I_{s s}\left(\gamma_{k}\right.$ is the Kerr coupling constant), $\delta$ is the ellipticity of the pump wave, $\varepsilon=\tau_{R} / \tau_{E r}$ is the ratio of the round-trip time $\tau_{R}$ to the lifetime of Erbium ions at the first excited level $\tau_{E r}$; $\chi=\left(\sigma_{a}{ }^{(L)}+\sigma_{e}{ }^{(L)}\right) / \sigma_{a}{ }^{(L)}, \sigma_{a}{ }^{(L)}$ and $\sigma_{e}^{(L)}$ are absorption and emission cross sections at the lasing wavelength; $\Delta$ is the detuning of the lasing wavelength with respect to the maximum of the gain spectrum (normalized to the gain spectral width); $\mathrm{d}_{1}=\chi /\left(\pi\left(1+\Delta^{2}\right)\right)$; functions $f_{i}(i=1,2,3)$ are related to the angular distribution of the dipole moments of the excited ions $n(\theta)$ expanded into a Fourier series $[17,18]$ :

$$
n(\theta)=\frac{n_{0}}{2}+\sum_{k=1}^{\infty} n_{1 k} \cos (k \theta)+\sum_{k=1}^{\infty} n_{2 k} \sin (k \theta), f_{1}=\left(\chi \frac{n_{0}}{2}-1\right)+\chi \frac{n_{12}}{2}, f_{2}=\left(\chi \frac{n_{0}}{2}-1\right)-\chi \frac{n_{12}}{2}, f_{3}=\chi \frac{n_{22}}{2} .
$$

The Eq. (3) presents the approximation where the dipole moments of the absorption and emission transitions for Erbium-doped silica are located in the plane orthogonal to the direction of the light propagation. This approximation allows deriving the finite dimension system presented by Eqs. (2) where only $n_{0}, n_{12}$, and $n_{21}$ components contribute to the vector dynamics. Using the more general assumption of the 3D orientation distribution of the dipole orientations [19-22] result in infinite dimension system with the more complex expansion of the angular distribution of the dipole moments into series of the Wigner's $D_{m n}^{l}$ or spherical $Y_{m n}$ functions [22].

In Eqs. 2, the absorption dynamics in CNT is neglected that holds because the saturable absorber relaxation time $\tau_{a} \sim 300 f s$ is smaller than the pulse width $T_{p} \sim 600$ fs. The four-level system describing absorption and emission in $\mathrm{Er}^{3+}$ ion is reduced to the two-level system here that is justified for pump powers used in experiments, namely $\mathrm{I}_{\mathrm{p}}<200 \mathrm{~mW}$ [23-25]. Sergeyev et al. have demonstrated that migration assisted upconversion (MAUP) in high concentration Erbium-doped fiber suppresses the first excited level lifetime more than 10 times [23-25]. To account this suppression, we mimic MAUP in Eqs. 2 in terms of decreased lifetime at the first excited level [17, 18]. Unlike previously used models based on either coupled nonlinear Schrödinger or Ginzburg-Landau equations [13, 14, 26-30], Eqs. 2 account for the vector nature of the interaction between an optical field and an erbium doped active medium, slow relaxation dynamics of erbium ions, linear birefringence in fiber and light induced anisotropy caused by elliptically polarized pump light. Thus, the model based on Eqs. 2 goes beyond the limitations of the previously used models and so leads to new types of vector solitons including brightdark rogue waves that have been found theoretically and experimentally. 


\section{WILEY-VCH}

To illustrate the interplay between in-cavity birefringence and the ellipticity of the pump wave, we solve Eqs. (2) numerically by using parameters values quite close to the experimental ones: $\alpha_{1}=21.53$, $\alpha_{0}=0.136, \alpha_{s}=1.8 \cdot 10^{-5}, \alpha_{2}=2.533, \chi=2.3, \Delta=0.1, I_{p}=75, \gamma=2 x 10^{-6}, \varepsilon=10^{-4}$. The dynamics was calculated for $660 \mathrm{~K}$ round-trips $(20 \mathrm{~ms})$. The results are shown in Figs. 6.
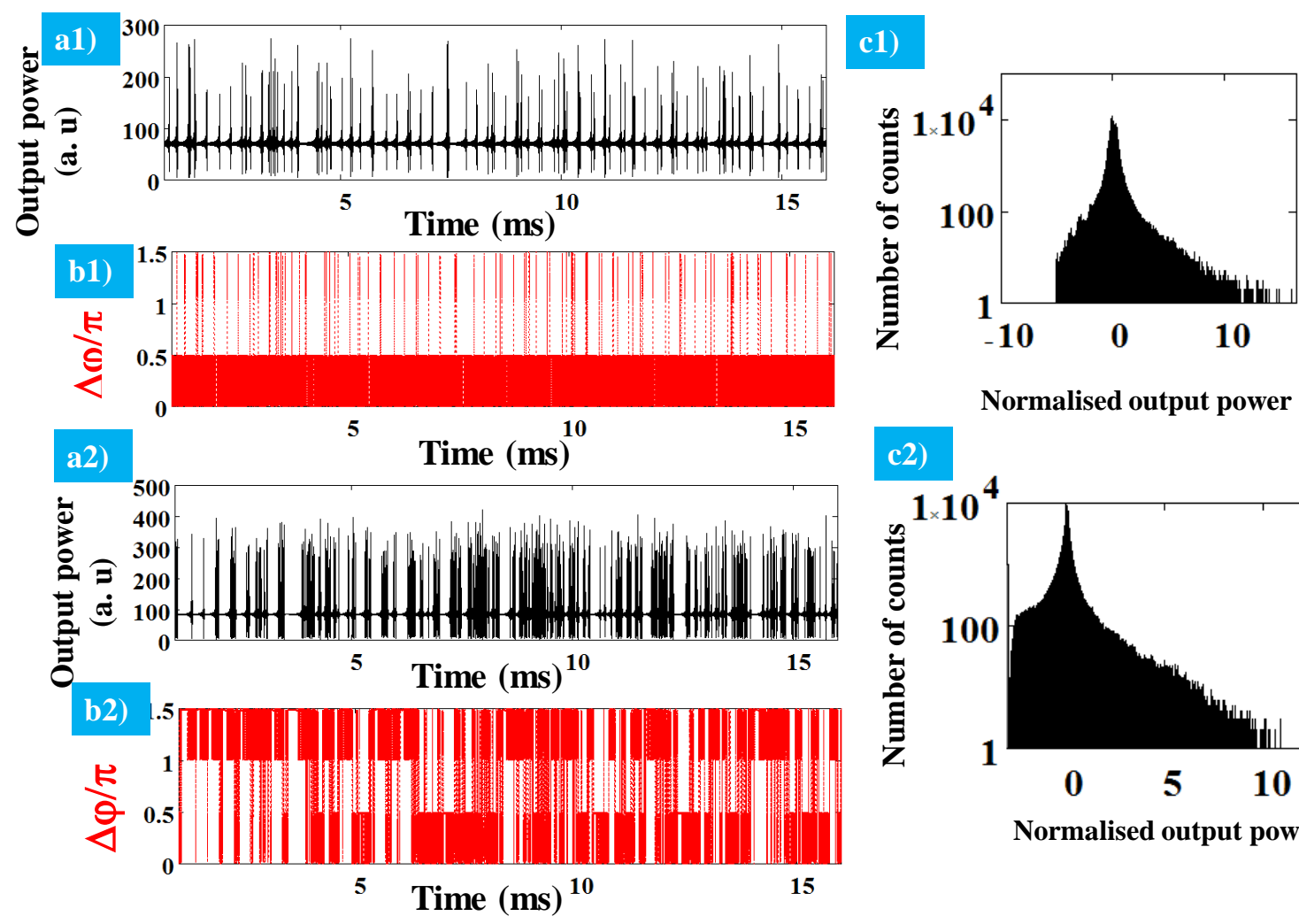

Normalised output power

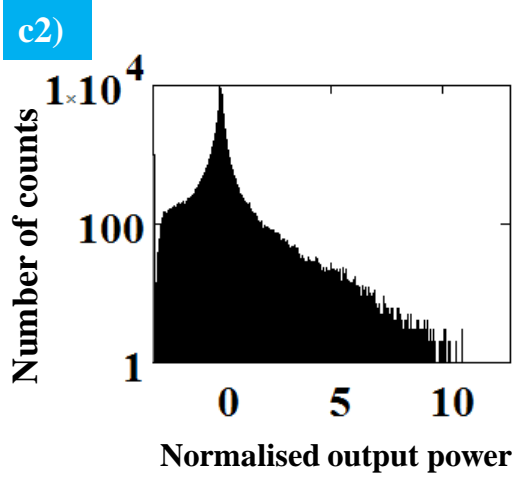

Figure 6 Laser dynamics averaged over the roundtrip time. Dynamics of the output power (a1, a2) and the phase difference (b1, b2) averaged over the pulse width; c1) and c2) corresponding probability distribution histograms (the output power I is normalized as shown in Fig.1). Parameters: $\alpha_{l}=21.53$, $\alpha_{0}=0.136, \alpha_{s}=1.8 \cdot 10^{-5}, \alpha_{2}=2.533, \chi=2.3, \Delta=0.1, \gamma=2 \times 10^{-6}, \varepsilon=10^{-4}$; a1), b1) the normalized pump power $I_{p}=75$; anisotropy of the pump wave $\delta=0.85$ (elliptically polarized pump), normalized birefringence strength $\beta=\pi \cdot 10^{-2}$; a2), b2) $I_{p}=90, \delta=0.94$ (elliptically polarized pump), $\beta=1.26 \pi \cdot 10^{-2}$.

As follows from Fig. 6 (a1, a2), the dynamics for the elliptically polarized pump takes the form of the chaotic oscillations with the probability distribution histogram satisfying rogue waves criteria. Lowpass filtering of the theoretical data over 30 round trips corresponds to the experimental conditions of the vector dynamics characterization based on a polarimeter with $1 \mathrm{MHz}$ resolution (Fig. 3).

The obtained results shown in Fig. 7 are in a good correspondence to the experimental data shown in Figs. 3. The anomalous spikes in the output power (Fig. 7 (a1), black) correspond to the phase difference jumps in $\pi$ (Fig. 7 (a1), red), i.e. transitions between orthogonally polarized SOPs, as follows from Fig. 7 (b1). The probability distribution diagrams for the total power $\mathrm{I}=\mathrm{I}_{\mathrm{x}}+\mathrm{I}_{\mathrm{y}}$ (Fig.7 (c1)) showing the 


\section{WILEY-VCH}

presence the bright-dark RWs corresponds to the histograms shown for $\mathrm{P}=26.4 \mathrm{~mW}$ in polarimetry experiments (Fig. 3). The results shown in Fig. 7 (a2-c2) demonstrates that low-pass filtering RWs results in suppression of RWs the same as it is demonstrated in the experiment (Fig. 3 (a2-c2)).

\section{a1)}

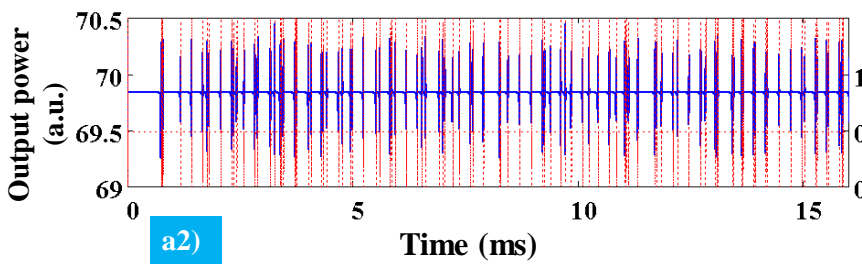

a2)

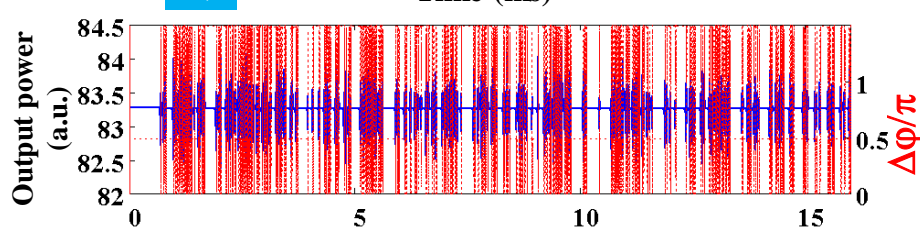

Time (ms)
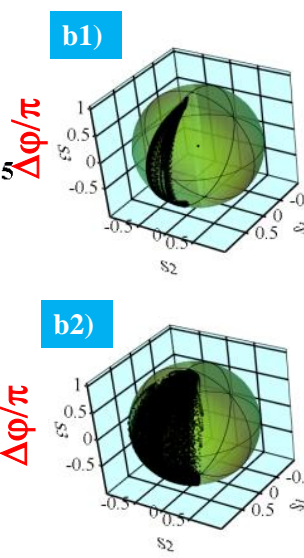

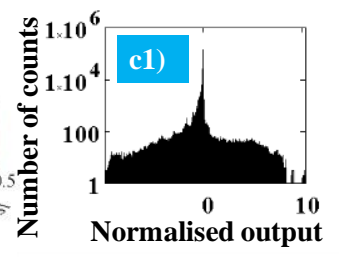

power

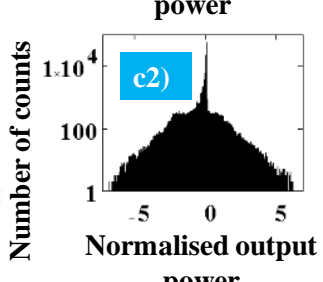

power

Figure 7 Dynamics after low-pass filtering with a Hanning window with transmission spectrum $\left(T(f)=\left(1+\cos \left(\pi f / f_{c}\right)\right) / 2, f f_{c}=1 M H z\right)$. a1 $)$, a2) dynamics of the output powers $\mathrm{I}=\mathrm{I}_{\mathrm{x}}+\mathrm{I}_{\mathrm{y}}$ (blue) and the phase difference $\Delta \phi$ (red); b1), b2) trajectories on the Poincaré sphere; c1), c2) Probability distribution histograms for the total output power $\mathrm{I}=\mathrm{I}_{\mathrm{x}}+\mathrm{I}_{\mathrm{y}}$. The output power $\mathrm{I}$ is normalized as shown in Fig.2 Parameters: $I_{p}=75 ; \alpha_{1}=21.53, \alpha_{0}=0.136, \alpha_{s}=1.8 \cdot 10^{-5}, \alpha_{2}=2.533, \chi=2.3, \Delta=0.1, \gamma=2 x 10^{-6}, \varepsilon=10^{-4}$; a1-c1) $\delta=0.85$ (elliptically polarized pump), $\beta=1.1 \pi \cdot 10^{-2} ; \mathrm{a} 2$ ) - c2) $I_{p}=90 ; \delta=0.94$ (elliptically polarized pump), $\beta=1.26 \pi \cdot 10^{-2}$.
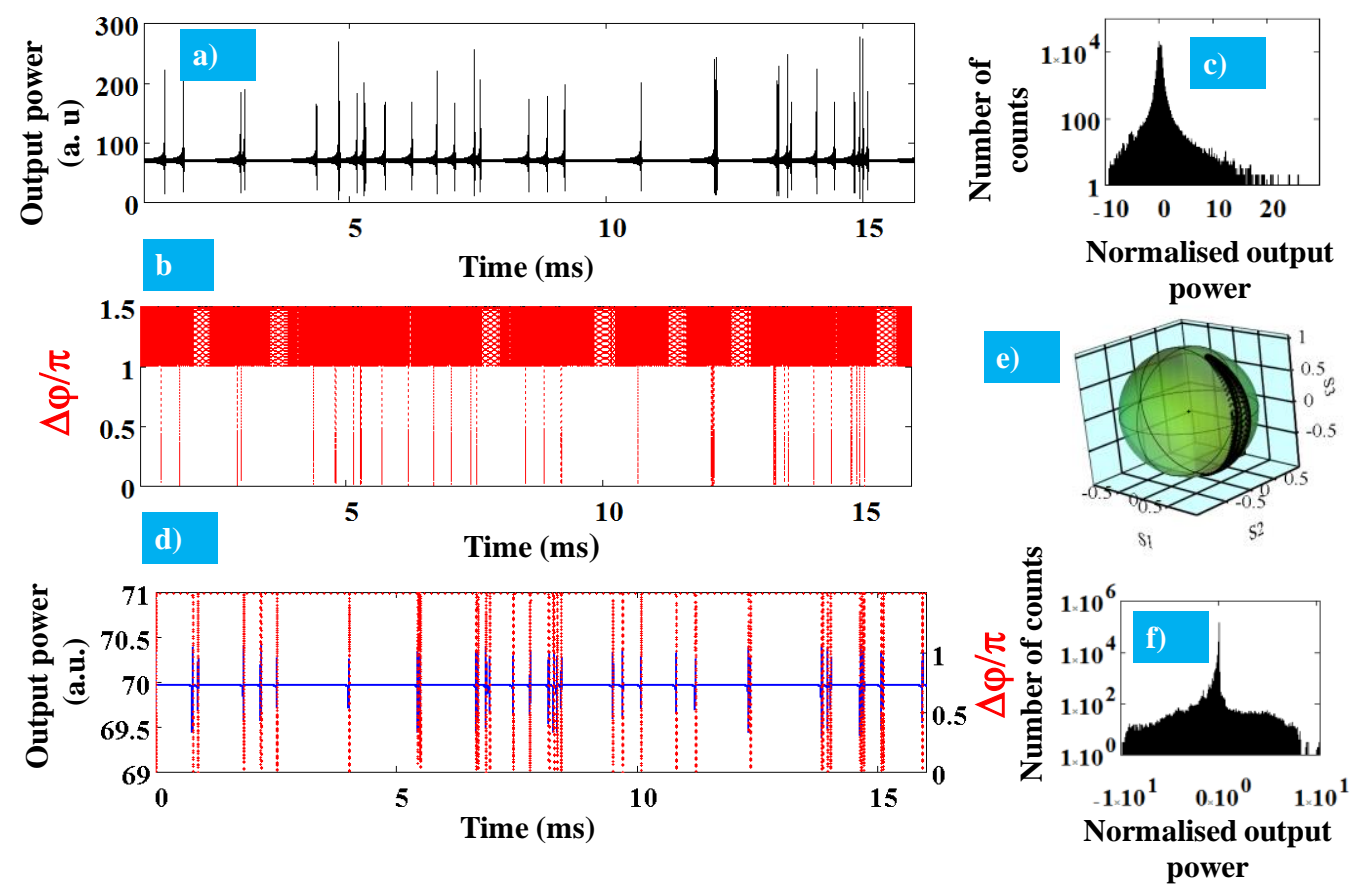

Figure 8 Laser dynamics averaged over the roundtrip time and after low pass filtering. Dynamics before (a-c) and after (d-f) low-pass filtering with a Hanning window with transmission spectrum $\left(T(f)=\left(1+\cos \left(\pi f / f_{c}\right)\right) / 2, f \leq f_{c}=1 M H z\right.$. a) Dynamics of the output power; b) dynamics of the phase difference $\Delta \phi$; c) probability distribution histograms (the output power I is normalized as shown in 


\section{WILEY-VCH}

Fig.2); d) dynamics of the output powers $\mathrm{I}=\mathrm{I}_{\mathrm{x}}+\mathrm{I}_{\mathrm{y}}$ (blue) and the phase difference $\Delta \phi$ (red); e) trajectories on the Poincaré sphere; f) Probability distribution histogram for the total output power (output power is normalized as shown in Fig.2). Parameters: $I_{p}=76 ; \alpha_{1}=21.53, \alpha_{0}=0.136, \alpha_{s}=1.8 \cdot 10^{-5}, \alpha_{2}=2.533$, $\chi=2.3, \Delta=0.1, \gamma=2 x 10^{-6}, \varepsilon=10^{-4}$; a-f) $\delta=0.84$ (elliptically polarized pump), $\beta=1.1 \pi \cdot 10^{-2}$.

The further tuning of the pump power, birefringence and the ellipticity of the pump wave result in the emergence of the bright-dark rogue waves as shown in Fig. 8 (a-f). Results of the data averaging over 30 round-trips (1 MHz low-pass filter) are shown in Fig. 8 (d-f). The obtained results are shown in Fig. 8 are in a good correspondence to the experimental data shown in Figs. 5. The anomalous spikes-dips in the output power (Fig. 8 (a, b, d)) coincides with the phase difference jumps in $\pi$ (Fig. 8 (b, d)), i.e. transitions between orthogonally polarized SOPs (Fig. 8 (e)). Probability distribution diagram for the total power $\mathrm{I}=\mathrm{I}_{\mathrm{x}}+\mathrm{I}_{\mathrm{y}}$ (Fig.8 (f)) is showing the presence the dark-bright RWs. Unlike this, only bright rogue waves are present in the experimental polarimetry data shown in Fig. 5 (c).

\section{Discussion: rogue waves emergence as a results of a desynchronization of coupled oscillators}

To explain the emergence of the RWs at the slow time scale, we attract a model of nonlinear coupled oscillators, which are widely used to study different synchronization scenarios from the phase locking to the chaotic phase drift in different context ranging from biomedicine to the laser physics [31-34]. As follows from Eqs. (3), the equation for the phase difference $\Delta \phi$ is a further generalization of Adler equation [31-34] :

$$
\frac{d \Delta \phi}{d t}=\Delta \Omega+K_{N L} \sin ^{2}(\Delta \phi)-K \sin (\Delta \phi-\alpha)
$$

Here $\Delta \Omega$ is a frequencies shift that depends on birefringence in the laser cavity comprising two parts, viz. birefringence of the passive fiber combined with birefringence induced by the in-cavity polarization controller and birefringence caused by polarization hole burning in the active fiber; $\alpha$ is the phase shift depending on the difference of the output powers $\mathrm{I}_{\mathrm{x}}-\mathrm{I}_{\mathrm{y}}$. Eq. (4) is accounting for coherent coupling of polarization modes ( $K$ coefficient) and coupling based on the Kerr effects $\left(K_{N L}\right)$. The frequency 


\section{WILEY-VCH}

difference $\Delta \Omega$, phase shift $\alpha$ and coupling coefficients $\mathrm{K}, \mathrm{K}_{\mathrm{NL}}$ depend on the $I_{x}$ and $I_{y}$ that means we have a new class of coupled oscillators with the time-dependent coupling strength [31-34].

It has been shown recently for such systems that by tuning the coupling strength and the oscillators' frequency difference, it is possible to tune the phase dynamics from the phase locking to the phase oscillations and chaotic phase slips including RWs [31-34]. In our case, the orthogonally polarized SOPs can be considered as coupled oscillators where the frequency difference depends on the birefringent strength and the coupling strength is a function of the ellipticity and the power of the pump wave [31]. Thus, adjustment of the pump wave and in-cavity polarization controllers (POC1 and POC2 in Fig. 1) and the pump power result in tuning the frequency difference and the coupling strength. As follows from Eq. (4), the condition for synchronization $(d \Delta \phi / d t=0)$ takes the form $|\Delta \Omega|<|\mathrm{K}|$. The results shown in Figs. 9 demonstrate how the adjustment of the birefringence strength, the ellipticity of the pump wave and the pump power changes the coupling strength and the frequency difference. The adjustment vary the system dynamics from the synchronization to desynchronization taking the form of the periodic and the chaotic phase difference oscillations leading to the rogue waves emergence (Figs. 5-8). 


\section{WILEY-VCH}
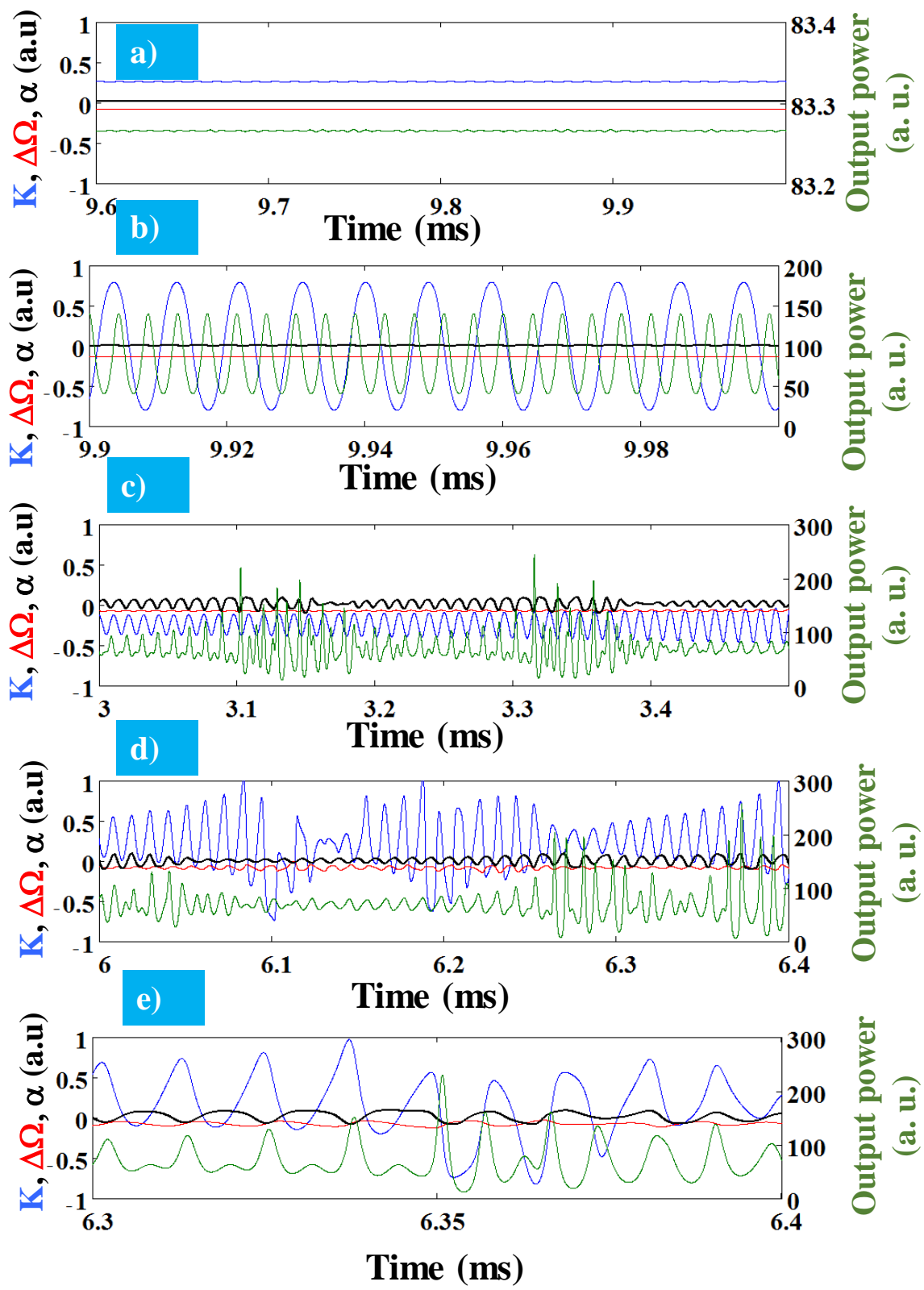

Figure 9 Different synchronization scenarios of the orthogonal linearly polarized modes. Synchronization (a) and desynchronization of the coupled polarization modes leading to the output power oscillations (b) and rogue waves (c-e). Output power I, the phase difference $\Delta \phi$, coupling coefficient $K$, phase difference $\Delta \Omega$, phase shift $\alpha$ as a function of time. Parameters: $\alpha_{1}=21.53, \alpha_{0}=0.136, \alpha_{s}=1.8 \cdot 10^{-5}, \alpha_{2}=2.533, \chi=2.3, \Delta=0.1, \gamma=2 \cdot 10^{-6}, \varepsilon=10^{-4}$; a) $\delta=0.8, \beta=\pi \cdot 10^{-2}$; b) $\delta=0.94, \beta=2 \pi \cdot 10^{-2}$; c) $\mathrm{I}_{\mathrm{p}}=75 ; \delta=0.85, \beta=1.1 \pi \cdot 10^{-2}$; d) $\mathrm{I}_{\mathrm{p}}=90 ; \delta=0.94$ $\beta=1.26 \pi \cdot 10^{-2}$; e) $\mathrm{I}_{\mathrm{p}}=76 ; \delta=0.84, \beta=1.1 \pi \cdot 10^{-2}$.

\section{Conclusion}

In summary, we report on experimental and theoretical data demonstrating a new type of the vector rogue waves resulting from the interaction between the orthogonal SOPs in an Er-doped mode-locked fiber laser with a saturable absorber. By tuning in-cavity and the pump wave polarization controllers, we adjust the coupling between SOPs and so provide conditions for 


\section{WILEY-VCH}

the appearance of the bright-dark rogue waves driven by the SOPs desynchronization. Such mechanism has been well justified based on a new vector model of mode-locked fiber laser which is different from the models demonstrating rogue waves in the form of the soliton rain [8-12] and well-known vector Peregrine (rational) solutions [13, 14], along with freak wave solutions having both exponential and rational dependence on coordinates [13]. The developed presentation of the rogue waves emergence as desynchronization events in the system of coupled oscillations show a great potential for mapping conditions for rogue waves emergence and so for developing techniques for suppression rogue waves in different distributed systems ranging from the lasers to power grids and telecom networks.

Acknowledgements This work has been funded through Leverhulme Trust (Grant ref: RPG2014-304); National Science Foundation of China 61605107, the Young Eastern Scholar program (QD2015027) at Shanghai Institutions of Higher Learning and the "Young 1000 Talent Plan" program of China; H2020 Marie-Sklodowska-Curie IF scheme; Austrian Science Fund (FWF project P24916-N27); support from the Ministry of Higher Education, Sultanate of Oman. The authors thanks Dr. Nikita Tarasov and Dr. Srikanth Sugavanam for fruit spatio-temporal intensity dynamics discussion.

Received: ((will be filled in by the editorial staff))

Revised: ((will be filled in by the editorial staff))

Published online: ((will be filled in by the editorial staff))

Keywords: rogue waves, coupled oscillators, mode-locked lasers, polarization

\section{References}

1. C. Kharif, E. Pelinovsky, A. Slunyaev, Rogue Waves in the Ocean. (Springer, Heidelberg, 2009).

2. Y. Zhen-Ya, Commun. Theor. Phys. 54, 947-949 (2010).

3. M. Onorato, S. Residori, U. Bortolozzo, A. Montina, F.T. Arecchi, Phys. Rep. 528, 47-89 (2013).

4. D. R. Solli, C. Ropers, P. Koonath, B. Jalali, Nature 450, 1054-1057 (2007).

5. Z. Liu, S. Zhang, F. W. Wise, Opt. Lett. 40, 1366-1369 (2015).

6. L. Gao, T. Zhu, S. Wabnitz, M. Liu, W. Huang, Optical polarization rogue waves in fiber laser. In Conference on Lasers and Electro-Optics, OSA Technical Digest (online) (Optical Society of America), https://doi.org/10.1364/CLEO_QELS. FF2A.8 (2016). 


\section{WILEY-VCH}

7. A. F. J. Runge, C. Aguergaray, N. G. R. Broderick, M. Erkintalo, Opt. Lett. 39, 319-322 (2014).

8. N. Akhmediev, B. Kibler, F. Baronio, M. Belić, W. P. Zhong, Y. Zhang, W. Chang, J. M. Soto-Crespo, P. Vouzas, P. Grelu, and C. Lecaplain, Roadmap on optical rogue waves and extreme events. J. Opt. 18, 063001 (2016).

9. J. M. Dudley, F. Dias, M. Erkintalo, G. Genty, Nature Photon. 8, 755-764 (2014).

10. C. Lecaplain, Ph. Grelu, J. M. Soto-Crespo, N. Akhmediev, J. Opt. 15, 064005 (2013).

11. C. Lecaplain, Ph. Grelu, J. M. Soto-Crespo, N. Akhmediev, Phys. Rev. Lett. 108, 233901 (2012).

12. J. M. Soto-Crespo, Ph. Grelu, N. Akhmediev, Phys. Rev. E 84, 016604 (2011).

13. F. Baronio, A. Degasperis, M. Conforti, S. Wabnitz, Phys. Rev. Lett. 109, 044102 (2012).

14. S. H. Chen, Ph. Grelu, J. M. Crespo, Phys. Rev. E 89, 011201(R) (2014).

15. J. P. Gordon, Optics Lett. 8, 596-598 (1983).

16. F. M. Mitschke, L. F. Mollenauer, Optics Lett. 12, 355-357 (1987).

17. S. V. Sergeyev, C. Mou, E. G. Turitsyna, A. Rozhin, S. K. Turitsyn, K. Blow, Light: Science \& Appl. 3, e131 (2014).

18. S. V. Sergeyev, Phil. Trans. R. Soc. A 372, 20140006 (2014).

19. H. Zeghlache, A. A, Boulnois, Phys. Rev. A 52, 4229-4242 (1995).

20. R. Leners, G. Stéphan, Quantum Semiclass. Opt. 7, 757-794 (1995).

21. S. V. Sergeyev, Phys Rev A 59, 3909- 3917 (1999).

22. V. A. Gaisenok, G. G. Krylov, S.V. Sergeyev, Physica D 72, 161-165 (1994).

23. S. V. Sergeyev, Electron. Lett. 39, 511-512 (2003).

24. S. V. Sergeyev, S. Popov, D. Khoptyar, A. Friberg, D. Flavin, JOSA B 23, 1540-1543 (2006).

25. S. V. Sergeyev, S. Popov, A. T. Friberg, Opt. Lett. 30, 1258-1260 (2005).

26. J. W. Haus, G. Shaulov, E. A. Kuzin, J. Sanchez-Mondragon, Opt. Lett. 24, 376-378 (1999).

27. S. T. Cundiff, B. C. Collings, N. N. Akhmediev, J. M. Soto-Crespo, K. Bergman, W. H. Knox, Phys. Rev. Lett. 82, 3988-3991 (1999).

28. H. Zhang, D. Y. Tang, L. M. Zhao, H. Y. Tam, Opt. Lett. 33, 2317-2319 (2008).

29. H. Zhang, D. Y. Tang, L. M. Zhao, X. Wu, Phys. Rev. E. 80, 052302 (2009).

30. D. Y. Tang, H. Zhang, L. M. Zhao, X. Wu, Phys. Rev. Lett. 101, 153904 (2008).

31. S. V. Sergeyev, Optics Lett. 41, 4700-4703 (2016). 


\section{WILEY-VCH}

32. A. Pikovsky, M. Rosenblum, J. Kurths, Synchronization: A Universal Concept in Nonlinear Science. (Cambridge University Press, Cambridge 2001).

33. A. Arenas, A. Díaz-Guilera, J. Kurths, Y. Moreno, Ch. Zhou, Phys. Rep. 469, 93-153 (2008).

34. G. Ansmann, R. Karnatak, K. Lehnertz, U. Feudel, Phys. Rev. E 8, 052911 (2013). 